\title{
DETECTION OF TWO-PHASE FLOW PATTERNS IN A VERTICAL MINICHANNEL USING THE RECURRENCE QUANTIFICATION ANALYSIS
}

\author{
*Romuald MOSDORF, *Grzegorz GÓRSKI \\ *Faculty of Mechanical Engineering, Bialystok University of Technology, Wiejska 45 C, 15-351 Bialystok, Poland \\ r.mosdorf@pb.edu.pl, grzesgor@o2.pl
}

received 6 October 2014, revised 20 July 2015, accepted 22 July 2015

\begin{abstract}
The two-phase flow (water-air) occurring in square minichannel $(3 \times 3 \mathrm{~mm})$ has been analysed. In the minichannel it has been observed: bubbly flow, flow of confined bubbles, flow of elongated bubbles, slug flow and semi-annular flow. The time series recorded by laser-phototransistor sensor was analysed using the recurrence quantification analysis. The two coefficients:Recurrence rate (RR) and Determinism (DET) have been used for identification of differences between the dynamics of two-phase flow patterns. The algorithm which has been used normalizes the analysed time series before calculating the recurrence plots. Therefore in analysis the quantitative signal characteristicswas neglected. Despite of the neglect of quantitative signal characteristics the analysis of its dynamics (chart of DET vs. RR) allows to identify the two-phase flow patterns. This confirms that this type of analysis can be used to identify the two-phase flow patterns in minichannels.
\end{abstract}

Key words:Two-Phase Flow, Recurrence Plot, Recurrence Quantification Analysis

\section{INTRODUCTION}

The identification of flow patterns in minichannels often depends on the subjective evaluation of the observer and used experimental technique (Zhao and Rezkallah,1993; Wongwises and Pipathattakul, 2006; Chen et al., 2006). Wang et. al in the paper (Wang et al., 2010) show that the non-linear analysis (Lempel-Ziv complexity and approximate entropy) of data from mini-conductance probe array and vertical multielectrode array conductance sensorenables the identification of flow patterns of the oil-gas-water mixture. The results of non-linear analysis of temperature and pressure fluctuations in microchannels are discussed by Mosdorf et.al in the paper (Mosdorf et al., 2005). The processes responsible for the appearance of pressure and temperature chaotic oscillations such as: nucleation, stability of bubbly flow, vapour core stability and vapour-phase flow stability are discussed. In the paper (Wang et al., 2003) it has been used the non-linear analysis of the pressure fluctuations to identify the flow patterns of air in water.Three kinds of flow patterns including bubble flow, churn flow and annular flow in the inlet tube were investigated by detecting time series of differential pressure. Hurst exponent, largest Lyapunov exponent and correlation dimensions have been used.In the paper (Zong et al., 2010) it has been shown that the methods characterizing the non-linear dynamics of the flow of oil-water are useful for identification of the flow patterns and for assessment of the complexity of these patterns. The recurrence quantification analysis and chaotic attractor geometry morphological description have been used for signal from vertical multi-electrode array. The results show that the proposed method of data analysis describes the flow structure complexity. Jin et al. in the paper (Jin et al., 2003) show that the correlation dimension and Kolmogorov entropy are sufficient to identify the flow patterns. Faszczewski et al.,(2012) used the recurrence plot method to analyse the flow patterns in a vertical mini channel.It has been shown that this method allows us to determine the parameters which define the borders between flow patterns.

The subject of the research is the development of method for identifying the types of flow patterns which occur during the movement of the gas-liquid mixture in the vertical minichannel. Usually the non-linear methods of data analysis require the long time series for calculation of different coefficients which characterize the system dynamics. In the present paper the proposed method is based on the recurrence quantification analysis. Such method does not require the analysis of long time series. Therefore, it can be useful for implementation in the sensors which automatically can identify the flow pattern. The main aim of the paper was the assessment if the signal, which qualitatively estimates the presence of air inside the minichannel, can be used for two-phase flow pattern identification. The laser- phototransistor sensor has been used for qualitative assessment of the presence of bubbles or slugs in the minichannel.

\section{EXPERIMENTAL SETUP}

In Fig. 1 the schema of experimental stand is presented. Due to the size of the minichannel the obtaining the bubbly flow inside it requires the usage of a special generator of mini bubbles (8 - Fig. 1a). The proportional pressure regulator (Metal Work Regtronic with an accuracy of $1 \mathrm{kPa}$ ) was used to maintain the constant overpressure in the supply tank (10 - Fig. 1a) - the overpressure was $50 \mathrm{kPa}$. Flow patterns were recorded with using the Casio EX-F1 digital camera at 1200 fps (336 x 96 pixels). Pressure difference between the inlet and outlet of minichannel was measured using the silicon pressure sensor MPX12DP (range 0-10 kPa, sensitivity $5.5 \mathrm{mV} / \mathrm{kPa}$, response time $1 \mathrm{~ms}$, accuracy $\pm 0.05 \mathrm{kPa}$ ).

During the experiment it was analysed the data recorded for different flow patterns (water-air at $21^{\circ} \mathrm{C}$ ) in a square channel $3 \times 3 \mathrm{~mm}$ 


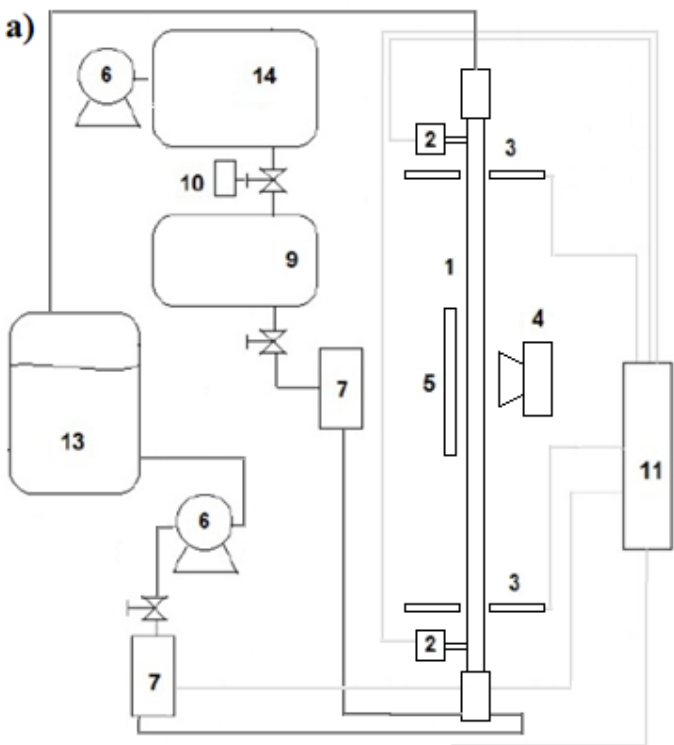

b)

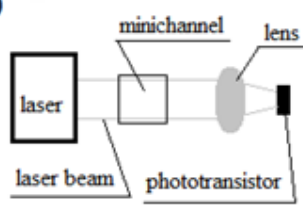

Fig. 1.Scheme of experimental stand. 1. minichannel, 2. pressure sensors (MPX12DP), 3. laser-phototransistor sensor. 4. Casio EX-FX1 camera, 5. lighting, 6 . pumps (air or water), 7. flow meters. 8. mini bubbles generator, 9 . air tank, 10. automatic valve to maintain a constant pressure in the tank 9,11 . data acquisition station (DT9800), 12. computer, 13. water tank, 14 air tank

The content of the minichannel (bubbles or liquid) has been qualitatively assessed using the laser-phototransistor sensor (3 - Fig. 1a). The schema of laser-phototransistor sensor is presented in Fig. 1b. The sensor consisted with a laser which generated the laser beam with a diameter of $3 \mathrm{~mm}$, the lens and phototransistor sensor placed in focal point of lens. Bubbles inside the minichannel bend the light which modifies the light intensity on the phototransistor. Sampling frequency was equal to $1 \mathrm{kHz}$.

\section{DATA ANALYSIS}

In Fig. 2 it has been shown the map of two phase flow patterns recorded during the experiment for different air and water flow rates. In the minichannel it has been observed: flow of isolated bubbles, flow of confined bubbles, flow of elongated bubbles, slug flow and semi-annular flow. Isolated bubbles have been observed for $q_{a}$ in the range from $0.001 \mathrm{l} / \mathrm{min}$ to $0.0254 \mathrm{l} / \mathrm{min}$. For $q_{a}$ equal to $0.0334 \mathrm{l} / \mathrm{min}$ and $0.0424 \mathrm{l} / \mathrm{min}$ the isolated bubbles are grouped. For $q_{a}$ equal to $0.1 \mathrm{l} / \mathrm{min}$ and $0.2 \mathrm{l} / \mathrm{min}$ the confined bubbles appear in the minichannel. Slugs flow appears for $q_{a}$ equal to $0.3 \mathrm{l} / \mathrm{min}$ and $0.4 \mathrm{l} / \mathrm{min}$ and semi-annular flow appears for $q_{a}=0.5 \mathrm{l} / \mathrm{min}$.

In Fig. 3 it has been shown the examples of recorded signals for different air volume flow rates $\left(q_{a}\right)$ and constant liquid volume flow rate $\left(q_{w}\right)$. When the channel is filled with water, the sensor generates a high voltage signal (about $3.5 \mathrm{~V}$ ). Such situation is visible in Fig.3a-h. Voltage drops below the maximum voltage level indicate that bubbles are in the minichannel. Such process is visible in Fig.3a and $b$ (small bubbles are in the channel). The appearance of the large bubbles in minichannel causes the significant voltage drops. Such process is visible in Fig.3c-h. When the bubbles group, then between the groups of bubbles the minichannel is filled with water without bubbles. Such process is visible in Fig.3e-h. When the confined bubble or slug occurs inside the minichannel, then the sensor generates a low voltage signal (about $3 \mathrm{~V}$ ). However, when the front of slug or confined bubble is passing through the laser beam, then the sensor voltage level drops to about 2 V. Such process is visible in Fig. 3i, j. When the minichannel is filled in with long slugs, then the sensor generates the signal shown in Fig.1k-m. In this case the signal fluctuations are caused by the slug diameter fluctuations. The above properties of laser - phototransistor sensors allow use the signal from sensors for qualitative assessment of the presence of bubbles or slugs in the minichannel.

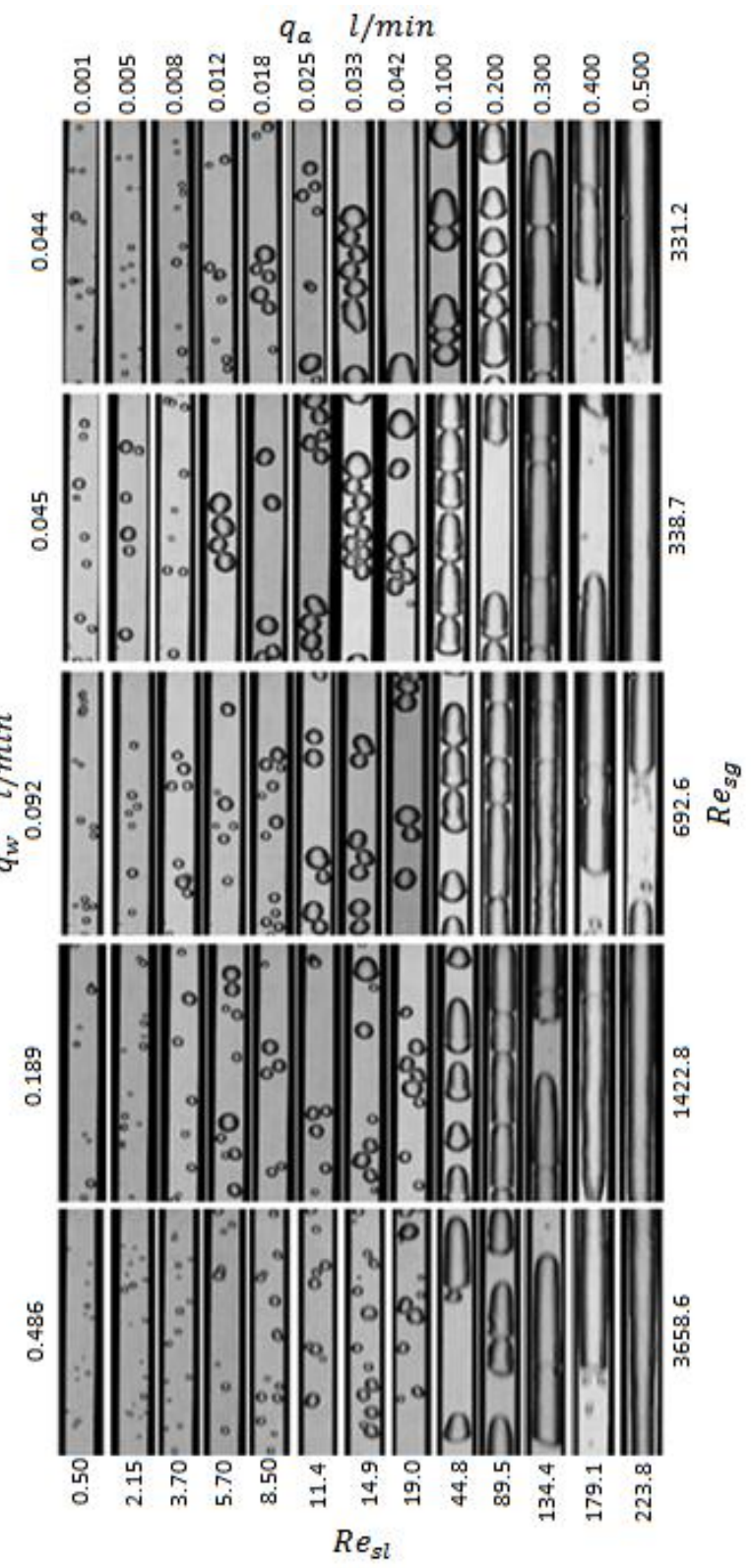

Fig. 2.The map of two phase flow patterns recorded during the experiment for different air and water flow rates 
The identification of flow patterns in minichannels often depends on the subjective evaluation of the observer and used experimental technique. For parameters characterizing the transition between flow patterns the two phase flow is usually unsteady. In such situation the criteria based on average values of the various parameters are not suitable for identification the border between flow patterns. Fig 4 presents the mean values of recorded data for different water flow rates. Non-linear character of function $\bar{x}\left(q_{a}\right)$ makes impossible the identification of the two phase flow pattern in minichannel based on the sensor data. The same values of $\bar{x}$ occur for different flow patterns. Therefore, the flow pattern identification requires the analysis of signal dynamics.
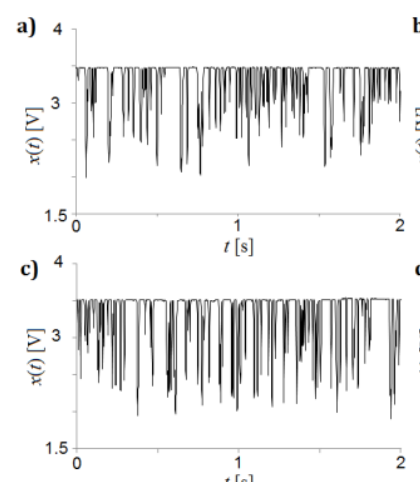

b) 4
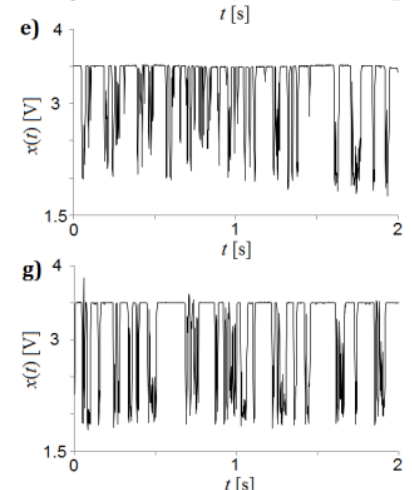

h) ${ }^{4}$

d) ${ }^{4}$

$\stackrel{1}{[}[\mathrm{s}]$
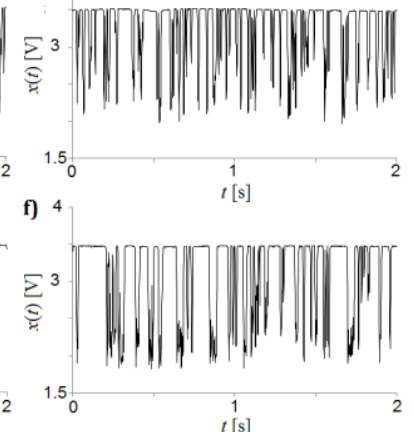

h) ${ }^{4}$
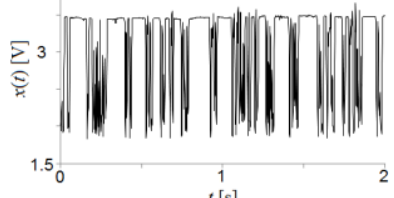

i) 5
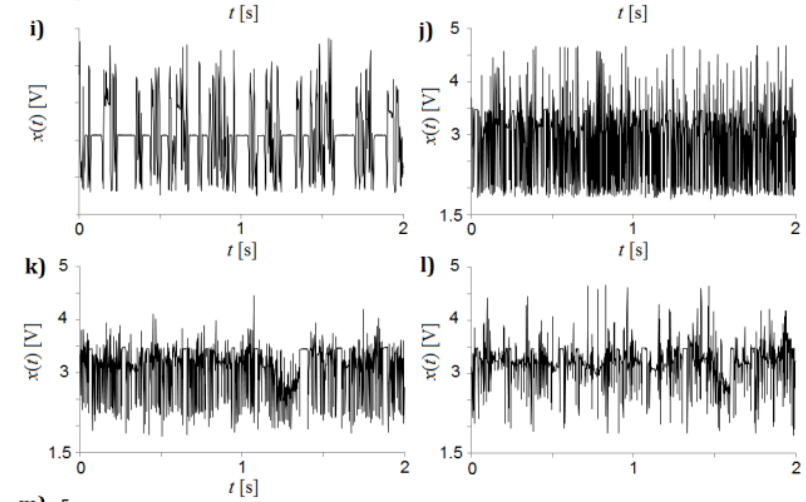

5

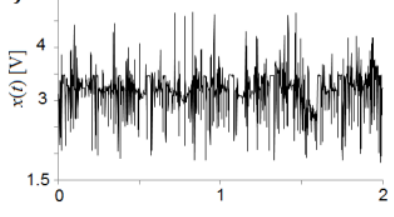

m) 5

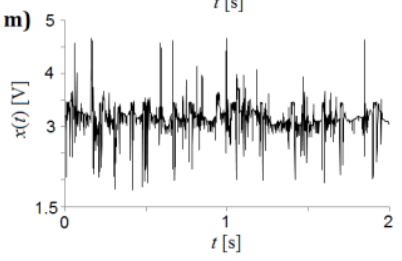

Fig. 3.Example of time series of pressure drop recorded during the experiment for $q_{w}=0.044 \mathrm{l} / \mathrm{min}$. a) $q_{a}=0.001 \mathrm{l} / \mathrm{min}$,

b) $q_{a}=0.00478 \mathrm{l} / \mathrm{min}$, c) $q_{a}=0.00827 \mathrm{l} / \mathrm{min} \mathrm{d}$ ) $q_{a}=0.0127 \mathrm{l} / \mathrm{min}$.

e) $q_{a}=0.0188 \mathrm{l} / \mathrm{min}$, f) $q_{a}=0.0254 \mathrm{l} / \mathrm{min}$, g) $q_{a}=0.0334 \mathrm{l} / \mathrm{min}$

h) $q_{a}=0.0424 \mathrm{l} / \mathrm{min}$. i) $q_{a}=0.1 \mathrm{l} / \mathrm{min}$, j) $\left.q_{a}=0.2 \mathrm{l} / \mathrm{min}, \mathrm{k}\right) q_{a}=0.3$

$\left.\mathrm{l} / \mathrm{min} \mathrm{I)} q_{a}=0.4 \mathrm{l} / \mathrm{min} . \mathrm{m}\right) q_{a}=0.5 \mathrm{l} / \mathrm{min}$

\section{RECURRENCE QUANTIFICATION ANALYSIS}

The analysis of attractor reconstructed from single time series of experimental data generated by non-linear dynamical system gives us information about the properties of the system such as system complexity and its stability. In non-linear analysis the reconstruction of attractor in a certain embedding dimension is carried out using the stroboscope coordination (Marwan et al. 2007). In this method the subsequent co-ordinates of attractor points are calculated basing on the subsequent samples, between which the distance is equal to time delay. The time delay is a multiplication of time between the samples.

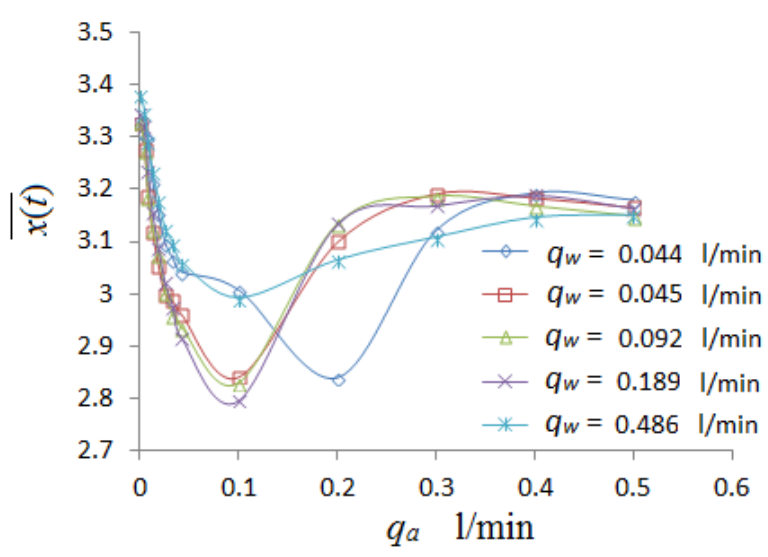

Fig. 4. The mean values of recorded data for different water flow rates

Recurrence plot is a technique of visualization of the recurrence of states $x_{i}$ in $m$-dimensional phase space. The states $x_{i}$ are points of attractor reconstructed in the $m$-dimensional phase space. The recurrence of states at time $i$ and at a different time $j$ is marked with black dots in the 2D plot, where both axes are time axes. The recurrence plot is defined as (Marwan et al. 2007):

$R_{i, j}=\Theta\left(\varepsilon-\left\|x_{i}-x_{j}\right\|\right), x_{i} \in \Re^{m}, i, j-1 \ldots N$

where $N$ is the number of considered states $x_{i}, \varepsilon$ is a threshold distance, $\|\cdot\|$ is a norm and $\Theta(\cdot)$ is the Heaviside function.

The researches based on the concept of recurrence plot are widely used for analyses of complex systems, especially for multiphase flow systems. In the paper (Zbilut, 2008) it has been shown that the Fourier transformation based on the recurrence plot can be used for the analysis of dynamics of higher dimensional spaces. Such analysis cannot be carried out by standard Fourier transformation. In the paper (Gaoet al.; 2013) the signals from multisector conductance sensor has been used for analysis of two different horizontal oil-water stratified flow patterns. The multivariate recurrence networks method and cross-transitivity has been used for recorded data. It has been shown that proposed method identifies the transition from a stable to an unstable state. In the paper (Yang al.,2015) the combustion instabilities in a diesel engine have been analysed. The combustion complexity has been analysed using the: delay-coordinate embedding, recurrence plot, Recurrence Quantification Analysis, correlation dimension, and the largest Lyapunov exponent. It has been found that the combustion system of diesel engine is a low-dimensional chaotic system. In the paper (Mosdorf et al., 2015) the pressure drop fluctuations in two-phase flow (water-air) in square minichannel $(3 \times 3 \mathrm{~mm})$ has been analysed. The two coefficients of Recurrence Quantification Analysis: recurrence period density entropy and 
transitivity have been used for identification of differences between the dynamics of two-phase flow patterns. It has been shown that the recurrence network analysis of dynamics of pressure drop fluctuations can be used for identify the two-phase flow patterns in minichannels.

In the paper, the time delay has been calculated based on the mutual information algorithm (Marwan et al., 2007). Embedding dimension has been estimated using the False Nearest Neighbours algorithm (Marwan et al., 2007).

The quantitative recurrence analysis generates the coefficients which describe the dynamics of two-phase flow patterns. Recurrence rate, $R R$, is a measure of the percentage of recurrence points in the recurrence plot. The value of $R R$ corresponds to the correlation sum. It is defined as follows (Marwan et al. 2007):

$R R=\frac{1}{N^{2}} \sum_{i, j=0}^{N} R_{i, j}$

The number of points which appears in the recurrence plot depends on the value of $\varepsilon$. Recurrence rate is a non-linear function of $\varepsilon$. In Fig. 5 it is presented the function $R R(\varepsilon)$ obtained for time series under consideration. Strong nonlinearity of function $R R(\varepsilon)$ is visible for the small value of $\varepsilon$. For higher value of $\varepsilon$ the nonlinearity becomes smaller (the function becomes quasi linear). It has been assumed that the lowest value of $\varepsilon$ in the linear part of function $R R(\varepsilon)$ is a proper value of $\varepsilon$ for the reconstruction of recurrence plot. For time series under consideration it is equal to 3 .

In Fig. 6 it has been shown the examples of recurrence plots of recorded data for different flow rates. The calculations have been made using the Matlab Toolbox (Marwan 2014). The used algorithm normalizes the analysed time series before calculating the recurrence plots. Therefore, in the analysis the quantitative signal characteristics was neglected.

In the recurrence plot a line parallel to main diagonal line occurs when a segment of the trajectory runs parallel to an another segment and the distance between trajectories is less than $\varepsilon$. The length of this diagonal line is determined by the duration of this phenomenon. A vertical (horizontal) line indicates a time in which a state does not change or changes very slowly. The diagonal lines (structures) periodically occurred in the recurrence plot are characteristic for periodic system (Marwan et al., 2007).

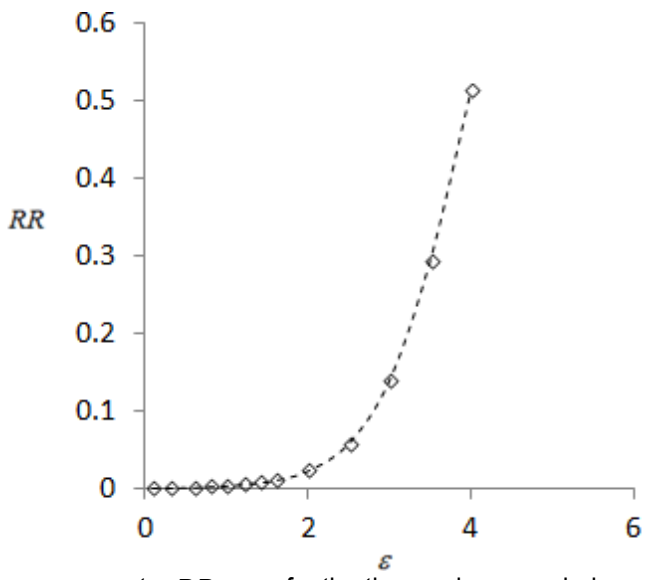

Fig. 5. Recurrence rate, $R R$, vs $\varepsilon$ for the time series recorded from the laser-phototransistor sensor for $q_{w}=0.059 \mathrm{l} / \mathrm{min}$, $q_{a}=0.0127 \mathrm{l} / \mathrm{min}$. The calculations have been made using the Matlab Toolbox (Marwan,2014) a)

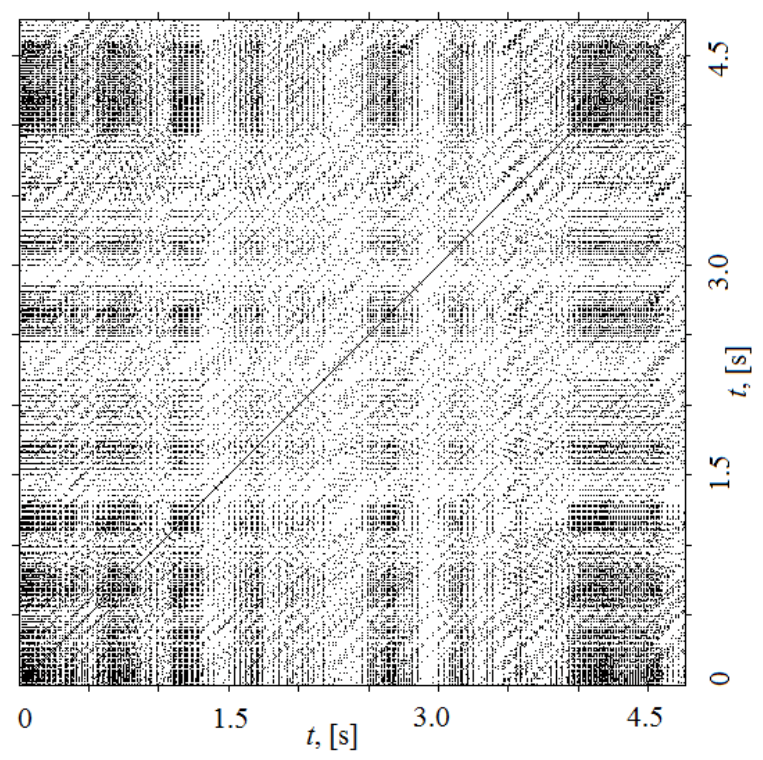

b)

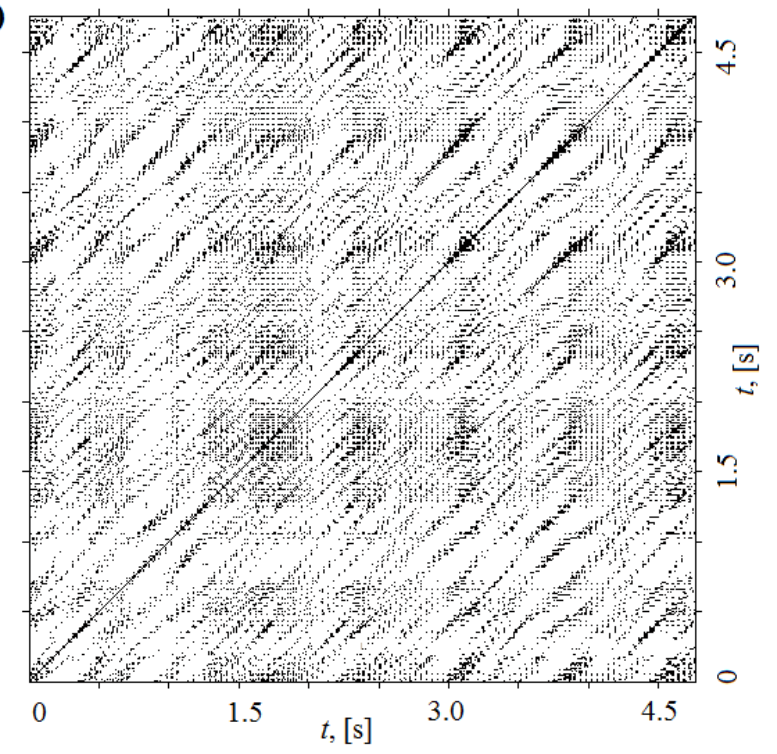

c)

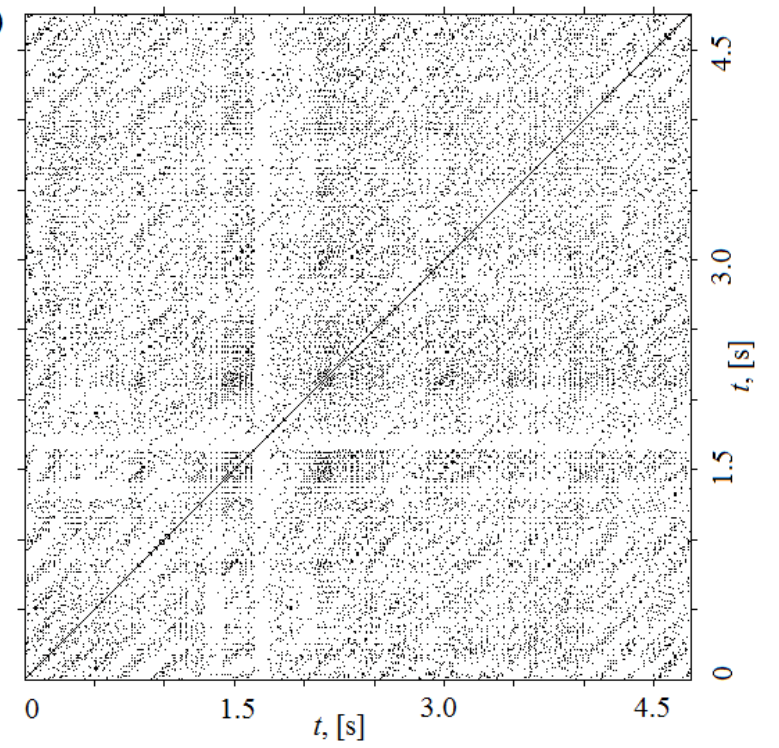

Fig. 6. Recurrence plots for embedding dimension 6 and time delay 1 $q_{w}=0.044 \mathrm{l} / \mathrm{min}$, a) $q_{a}=0.001 \mathrm{l} / \mathrm{min}$, b) $q_{a}=0.0424 \mathrm{l} / \mathrm{min}$, c) $q_{a}=0.1 \mathrm{l} / \mathrm{min}$. The calculations have been made using the Matlab Toolbox (Marwan,2014) 

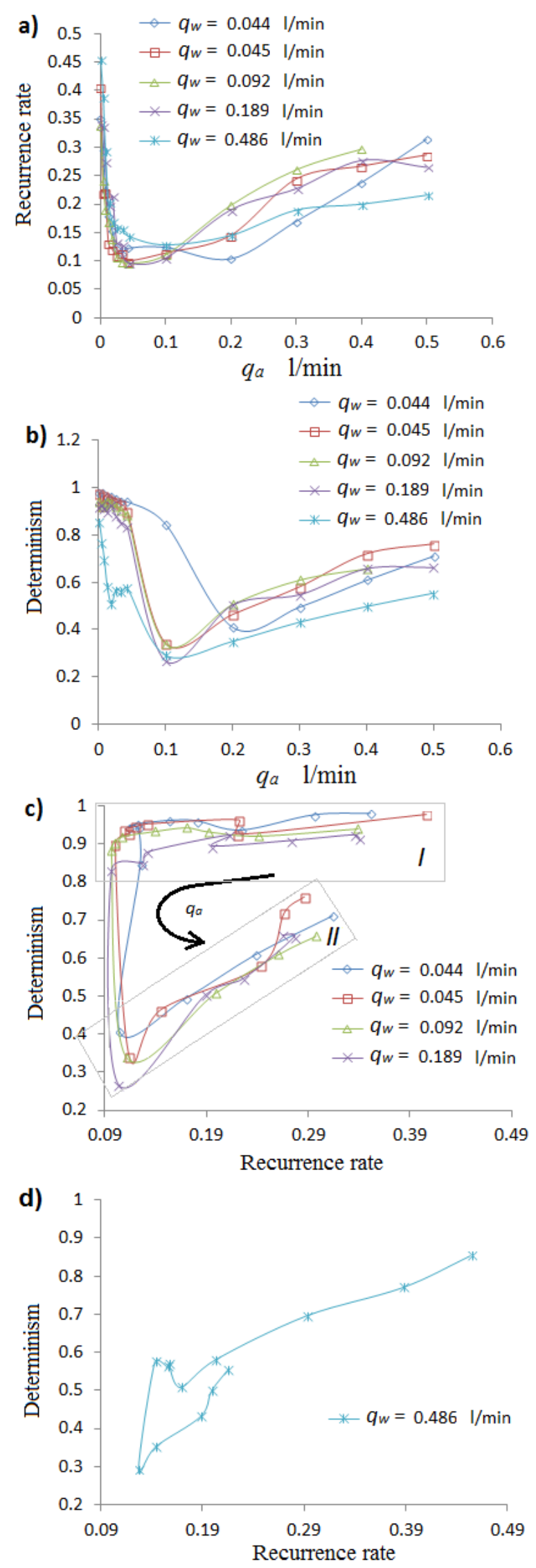

Fig. 7.Recurrence rate and Determinism for the different air and water flows. a) Recurrence rate. b) Determinism. c) Determinism vs. Recurrence rate. d) Determinism vs. Recurrence rate for $q_{w}=0.486 \mathrm{l} / \mathrm{min}$. The calculations have been made using the Matlab Toolbox (Marwan,2014)
The characteristics of diagonal lines are described by Determinism (DET) coefficient:

$D E T=\frac{\sum_{l=l \min }^{N} l P^{\varepsilon}(1)}{\sum_{i, j}^{N} R_{i, j}^{m, \varepsilon}}$

where $P(l)$ is the distribution of the lengths of diagonal structures and $N$ is the absolute number of diagonal lines;

The 13 coefficients characterizing the dynamics of the recorded signal were determined with using the Matlab Toolbox (Marwan;2014). The analysis of changes of those coefficients values together with flow pattern changes allows us to choose the two coefficients, whose values vary significantly over the considered range of parameters and unambiguously characterize two-phase flow patterns. These two coefficients $(R R, D E T)$ have been used for identification of differences between the dynamics of recorded data.

The functions of $R R\left(q_{a}\right)$ and $\operatorname{DET}\left(q_{a}\right)$ are shown in Fig.7a and b. In Fig.7c the chart of $D E T$ vs. $R R$ for $q_{a}$ in the range from $0.044 \mathrm{l} / \mathrm{min}$ to $0.189 \mathrm{l} / \mathrm{min}$ is presented. The lines indicate the order of points, black arrow indicates the direction of $q_{a}$ increase. The two rectangles marked with numbers $(I, I I)$ identify the two types of different flow patterns.

In the rectangle $I$ there are located the points which characterize the bubbly flow. In the right side of the rectangle $I$ there are located the points which characterize the bubbly flow with small isolated bubbles. In the left side of rectangle there are located points which are created by flow of grouped bubbles. In all cases under considerations the bubble diameters are less than the channel size.

In the rectangle $I I$ there are located the points characterizing the flow patterns where the bubbles fulfill the channel. Here there are located the points created by: confined and elongated bubbles flow, slug flow and semi-annular flow. In the left side of the rectangle $I I$ there are points characterising the flow of confined bubbles, but in the right side of the rectangle there are located points created by semi-annular flow.

The chart $D E T$ vs. $R R$ for $q_{w}=0.486 \mathrm{I} / \mathrm{min}$ is presented separately in Fig.7d because the points of this chart do not lie within the rectangles presented in Fig.7c. This is probably caused by too low sampling frequency for flow velocity for $q_{w}=0.486 \mathrm{I} / \mathrm{min}$. Therefore, we can conclude that for the water flow rates in the range from 0.044 to $0.189 \mathrm{l} / \mathrm{min}$ the chart $D E T$ vs. $R R$ (presented in Fig. 7c) can be used for two phase flow identification.

\section{CONCLUSIONS}

The proposed method for identifying the two-phase flow patterns is based on the analysis of the dynamics of the measurement signal.

The Recurrence Rate which has been used in the analysis is a measure of the probability of the recurrence. Such probability decreases with the increase of the air volume flow rate (Fig.7c). Such decrease is accompanied by increase of bubblediameters. For the slug flow the increase of air volume flow rate causes the increase of recurrence rate. It means that the number of recurrences increases.

The determinism (DET) is a measure of predictability of the system. For deterministic and periodic system, all points of $R P$ belong to the diagonal lines. For white noise all points of $R P$ are isolated points. Increase of the DET means that the system becomes more predictable. In bubble flow the predictability of the 
system does not change significantly (Fig.7c). The slug flow appearance in the minichannel rapidly decreases the predictability of the system (the length of the slugs and distance between them change chaotically). Further increase of the air volume flow rate makes the system more predictable (the length of the slugs and distance between them become more predictable). In the paper it has been shown that the simple laser- phototransistor sensor which can qualitatively assess the presence of bubbles or slugs in the minichannel can be used for identification of two phase flow patterns.

The RP analysis normalizes the signal so the average values of the signal do not affect the results of the analysis. Despite of the neglect of quantitative signal characteristics the qualitative analysis of its dynamics allows us to identify the two-phase flow patterns. This confirms that this type of analysis can be used to identify the two-phase flow patterns in minichannels in the relatively wide range of liquid and air flow rates. The final verification of the proposed method requires much larger number of analyses of different types of two-phase flows.

\section{REFERENCES}

1. Chen L., Tian Y.S., Karayiannis T.G. (2006), The effect of tube diameter on vertical two-phase flow regimes in small tubes, International Journal of Heat and Mass Transfer, vol. 49, 4220-4230.

2. Faszczewski M., Górski G., Mosdorf R. (2012), Applying recurrence plots to identify borders between two-phase flow patterns in vertical circular mini channel, Acta Mechanica et Automatica, Vol. 6, No.1, 31-36.

3. Jin N.D., X.B. Nie, Y.Y. Ren, Liu X. B.(2003), Characterization of oil/water two-phase flow patterns based on nonlinear time series analysis Flow Measurement and Instrumentation, Vol. 14, 169-175.

4. Zbilut J. P., Marwan N.(2008), The Wiener-Khinchin theorem and recurrence quantification, Physics Letters A 372, 6622-6626.

5. Marwan N., (2014), Cross Recurrence Plot Toolbox for Matlab, Ver. 5.15, Release 28.10, http://tocsy.pik-potsdam.de.
6. Marwan N., Romano M. C., Thiel M., Kurths J.. (2007), Recurrence Plots for the Analysis of Complex Systems, Physics Reports, 438 (5-6), 237-329.

7. Mosdorf R., Cheng P., Wu H.Y., Shoji M. (2005), Non-linear analyses of flow boiling in microchannels, International Journal of Heat and Mass Transfer, 48, 4667-4683.

8. Mosdorf R., Górski G. (2015), Detection of two-phase flow patterns using the recurrence network analysis of pressure drop fluctuations, International Communications in Heat and Mass Transfer, Vol.64, 14-20.

9. Wang S.F., Mosdorf R., Shoji M. (2003), Nonlinear analysis on fluctuation feature of two-phase flow through a T-junction, International Journal of Heat and Mass Transfer, 46, 1519-1528.

10. Wang Z.Y., JinN.D., GaoZ.K., ZongY.B., WangT. (2010), Nonlinear dynamical analysis of large diameter vertical upward oil-gas-water three-phase flow pattern characteristics, Chemical Engineering Science, 65, 5226-5236.

11. Wongwises S., Pipathattakul M. (2006), Flow pattern, pressure drop and void fraction of two-phase gas-liquid flow in an inclined narrow annular channel, Experimental Thermal and Fluid Science, 30, 345-354.

12. Yan-Bo Zong, Ning-De Jin, Zhen-Ya Wang, Zhong-Ke Gao, Chun Wang, (2010, Nonlinear dynamic analysis of large diameter inclined oil-water two phase flow pattern, International Journal of Multiphase Flow, 36, 166-183.

13. Yang L. P., Ding S. L., Litak G., Song E. Z., Ma X. Z. (2015), Identification and quantification analysis of nonlinear dynamics properties of combustion instability in a diesel engine, Chaos, 25(1), 013105

14. Zhao L., Rezkallah K.S. (1993), Gas-liquid flow patterns at microgravity condition, International Journal Multiphase Flow, 19, 751-763.

15. Zhong-Ke Gao, Xin-Wang Zhang, Ning-De Jin, Reik V. Donner, Norbert Marwan and Jürgen Kurths (2013), Recurrence networks from multivariate signals for uncovering dynamic transitions of horizontal oil-water stratified flows, Europhysics Letters, 103 (5), 1-7.

Acknowledgement: The project was funded by the National Science Centre, Poland - the number of decision: DEC-2013/09/B/ST8/02850 